\title{
Tail approximations to the density function in EVT
}

\author{
Jürg Hüsler • Deyuan Li
}

Received: 14 June 2006 / Revised: 10 August 2006 /

Accepted: 10 August 2006 / Published online: 28 October 2006

(C) Springer Science + Business Media, LLC 2006

\begin{abstract}
Let $X_{1}, X_{2}, \ldots, X_{n}$ be independent identically distributed random variables with common distribution function $F$, which is in the max domain of attraction of an extreme value distribution, i.e., there exist sequences $a_{n}>0$ and $b_{n} \in \mathbb{R}$ such that the limit of $P\left(a_{n}^{-1}\left(\max _{1 \leq i \leq n} X_{i}-b_{n}\right) \leq x\right)$ exists. Assume the density function $f$ (of $F$ ) exists. We obtain an uniformly weighted approximation to the tail density function $f$, and an uniformly weighted approximation to the tail density function of $P\left(a_{n}^{-1}\left(\max _{1 \leq i \leq n} X_{i}-b_{n}\right) \leq x\right)$ under some second order condition.
\end{abstract}

Keywords Tail approximation - Density function • Maximum • Extreme value distribution $\cdot$ Differentiable domain of attraction

AMS 2000 Subject Classifications $62 \mathrm{G} 32 \cdot 60 \mathrm{G} 70$

\section{Introduction}

Let $X_{1}, X_{2}, \ldots, X_{n}$ be independent identically distributed (i.i.d.) random variables (r.v.'s) with common distribution function (d.f.) $F$. Assume $F \in$

Partially supported by a grant of the Swiss National Science Foundation.

J. Hüsler $(\bowtie) \cdot$ D. Li

Department of Mathematical Statistics and Actuarial Science, University of Bern,

3012 Bern, Switzerland

e-mail: juerg.huesler@stat.unibe.ch 
$\mathcal{D}\left(G_{\gamma}\right)$ with $\gamma \in \mathbb{R}$, i.e., there exist sequences $a_{n}>0$ and $b_{n} \in \mathbb{R}$ such that as $n \rightarrow \infty$,

$$
P\left(\frac{\max _{1 \leq i \leq n} X_{i}-b_{n}}{a_{n}} \leq x\right)=F^{n}\left(a_{n} x+b_{n}\right) \rightarrow G_{\gamma}(x):=\exp \left(-(1+\gamma x)^{-1 / \gamma}\right)
$$

for all $x$ with $1+\gamma x>0$.

There exist many papers considering the uniform convergence of Eq. 1.1. For example, Smith (1982) discusses the uniform rates of convergence in Eq. 1.1, de Haan and Resnick (1996) give the exact rate of convergence in Eq. 1.1 under some second order condition. On the other hand, the extreme value condition Eq. 1.1 can also be rephrased in the following way:

$$
\lim _{n \rightarrow \infty} n \bar{F}\left(a_{n} x+b_{n}\right)=(1+\gamma x)^{-1 / \gamma}, \quad \text { for } 1+\gamma x>0 .
$$

Here $\bar{F}=1-F$. Drees et al. (2006) present a weighted approximation of $n \bar{F}\left(a_{n} x+b_{n}\right)$ under some second order condition. Based on this approximation, Dress et al. (2003) derive a weighted approximation of Eq. 1.1, which improves the result of de Haan and Resnick (1996).

Pickands (1986) defines the " $L$ times differentiable domain of attraction", where $L$ is a nonnegative integer. $F$ lies in the $L$ times differentiable domain of attraction, if and only if

$$
\lim _{n \rightarrow \infty} a_{n}^{l}\left(F^{n}\right)^{(l)}\left(a_{n} x+b_{n}\right)=G_{\gamma}^{(l)}(x), \quad \text { for } 1+\gamma x>0, l=0,1, \ldots, L,
$$

where $(l)$ denotes the $l$ th derivative of the function with respect to its argument. The necessary and sufficient conditions that, $F$ lies in the $L$ times differentiable domain of attraction for $L=1$ and $L=2$, are given in Pickands (1986). de Haan and Resnick (1982) dealt with the case $L=1$ and showed that under some conditions the density of the normalized maximum converges to the density of the limiting extreme value distribution in the $L_{p}$ metric.

Condition (1.3) is obviously stronger than Condition (1.1) if $L \geq 1$. In case of $L=1$, Eq. 1.3 implies not only Eq. 1.2 but also that the density function $f$ (of $F$ ) exists and

$$
\lim _{n \rightarrow \infty} n a_{n} f\left(a_{n} x+b_{n}\right)=(1+\gamma x)^{-1 / \gamma-1}, \quad \text { for } 1+\gamma x>0 .
$$

In this paper, we focus on the approximations of Eq. 1.4 and Eq. 1.3. We first derive a weighted approximation of $n a_{n} f\left(a_{n} x+b_{n}\right)$ (see Theorem 2.1 below), and then based on this approximation, we obtain a weighted approximation of Eq. 1.3 for $L=1$ (see Theorem 2.2 below).

Our results are necessary for certain applications in extreme value theory (EVT) as the following application in finance and economics. Gabaix and Laibson (2003) study the following problem in firm pricing. Suppose $X_{1}$, $X_{2}, \ldots, X_{n}$ are i.i.d. random variables with d.f. $F$, where $F \in \mathcal{D}\left(G_{0}\right)$ and $0<F(x)<1$ for all $x \in \mathbb{R}$. For any sequence $\left\{p_{i}\right\}_{i=1}^{n}$ satisfying $\left|p_{i}\right| \leq C<$ $\infty, i=1,2, . ., n$, define the demand function

$$
P_{n}:=P\left(X_{1}-p_{1} \geq \max _{2 \leq i \leq n}\left\{X_{i}-p_{i}\right\}\right) .
$$


Of course $P_{n}$ converges to zero as $n \rightarrow \infty$, and $P_{n}=1 / n$ if all $p_{i}$ 's are equal. But for general $p_{i}$ 's, how fast $P_{n}$ converges to zero? Hashorva and Hüsler (2000) and Rinott and Rotar (2001) approximate the rate of $P_{n}$ for normal distribution with particular choice of $p_{i}$ 's. Gabaix et al. (2003) mention a conjecture on the rate of $P_{n}$ but without rigorous proof. Note that

$$
\begin{aligned}
P_{n} & =\int_{-\infty}^{\infty} f(x) \prod_{i=2}^{n} F\left(x-p_{1}+p_{i}\right) \mathrm{d} x \\
& =\frac{1}{n} \int_{-\infty}^{\infty} n a_{n} f\left(a_{n}\left(x+p_{1} / a_{n}\right)+b_{n}\right) \prod_{i=2}^{n} F\left(a_{n}\left(x+p_{i} / a_{n}\right)+b_{n}\right) \mathrm{d} x .
\end{aligned}
$$

To approximate $P_{n}$, we approximate $a_{n} f\left(a_{n} x+b_{n}\right)$ and $F\left(a_{n} x+b_{n}\right)$ uniformly. The investigation of the mentioned conjecture is discussed in $\mathrm{Li}$ and de Vries (2006).

In this paper we present our main results in Section 2 with the proofs in Section 3. The results are assuming the following second order condition. We define $U(t):=F^{\leftarrow}(1-1 / t), t \geq 1$, and consider the conditions in de Haan and Resnick (1996):

$\left\{\begin{array}{l}\text { suppose } U \text { is twice differentiable, } U^{\prime} \text { is eventually positive, and the function } \\ A(t):=\frac{t U^{\prime \prime}(t)}{U^{\prime}(t)}-\gamma+1 \text { has constant sign near infinity and satisfies } \\ A(t) \rightarrow 0 \text { as } t \rightarrow \infty \text { and }|A| \in \mathrm{RV}(\rho) \text { with } \rho \leq 0 .\end{array}\right.$

We mention that Eq. 1.5 implies Eq. 1.3 with $L=2$. To show this, let $\tilde{U}(t)=$ $F^{\leftarrow}\left(1-e^{-t}\right), t \geq 0$. By Theorem 2.1 and Theorem 5.1 in Pickands (1986), it suffices to prove $\tilde{U}^{\prime \prime}(t) / \tilde{U}^{\prime}(t) \rightarrow c \in(-\infty, \infty)$ as $t \rightarrow \infty$. Note that $\tilde{U}^{\prime}(t)=$ $U^{\prime}\left(e^{t}\right) e^{t}$ and $\tilde{U}^{\prime \prime}(t)=U^{\prime \prime}\left(e^{t}\right) e^{2 t}+U^{\prime}\left(e^{t}\right) e^{t}$. So,

$$
\frac{\tilde{U}^{\prime \prime}(t)}{\tilde{U}^{\prime}(t)}=\frac{e^{t} U^{\prime \prime}\left(e^{t}\right)}{U^{\prime}\left(e^{t}\right)}+1 \rightarrow \gamma
$$

as $t \rightarrow \infty$ by Eq. 1.5. Thus relation 1.3 holds with $L=2$.

\section{Main Results}

Suppose Eq. 1.5 holds. Then by Theorem 2.1 in de Haan and Resnick (1996), it follows that

$$
U^{\prime}(t)=k t^{\gamma-1} \exp \left(\int_{1}^{t} \frac{A(u)}{u} \mathrm{~d} u\right)
$$

where $k>0$, and that, as $t \rightarrow \infty$,

$$
\frac{\frac{U^{\prime}(t x)}{U^{\prime}(t)}-x^{\gamma-1}}{A(t)} \rightarrow x^{\gamma-1} \frac{x^{\rho}-1}{\rho}, \quad \text { for } x>0 .
$$


We rewrite the convergence in Eq. 2.2. Define the function $K_{\gamma, \rho}$ by

$$
K_{\gamma, \rho}(x)= \begin{cases}\frac{\log ^{2} x}{2}, & \rho=0=\gamma, \\ \frac{x^{\gamma} \log x}{\gamma}, & \rho=0 \neq \gamma, \\ \frac{x^{\gamma+\rho}-1}{\gamma+\rho}, & \rho<0 .\end{cases}
$$

It is easy to see that there exist functions $\tilde{a}$ and $\tilde{A}$ such that as $t \rightarrow \infty$,

$$
\frac{\frac{U^{\prime}(t x)}{t^{-1} \tilde{a}(t)}-x^{\gamma-1}}{\tilde{A}(t)} \rightarrow \frac{\mathrm{d}}{\mathrm{d} x}\left(K_{\gamma, \rho}(x)\right)= \begin{cases}x^{-1} \log x, & \rho=0=\gamma, \\ x^{\gamma-1} \log x+\frac{x^{\gamma-1}}{\gamma}, & \rho=0 \neq \gamma, \\ x^{\gamma+\rho-1}, & \rho<0 .\end{cases}
$$

For example, in case of $\rho=0=\gamma$, let $\tilde{a}(t)=t U^{\prime}(t)$ and $\tilde{A}(t)=A(t)$; in case of $\rho=0 \neq \gamma$, let $\tilde{a}(t)=t U^{\prime}(t)(1-\tilde{A}(t) / \gamma)$ and $\tilde{A}(t)=A(t)$; in case of $\rho<0$, let $\tilde{a}(t)=t U^{\prime}(t)(1-\tilde{A}(t))$ and $\tilde{A}(t)=A(t) / \rho$. In the following we choose these particular functions $\tilde{a}$ and $\tilde{A}$.

The following proposition is a uniformly weighted convergence of Eq. 2.4, which is the key for deriving the tail approximation to the density function.

Proposition 2.1 Suppose Eq. 1.5 holds. Then there exists a function $a_{0}$ such that for each $\varepsilon>0$, there exists a $t_{\varepsilon}>0$ such that for all $t, t x>t_{\varepsilon}$

$$
x^{-(\gamma+\rho-1)} e^{-\varepsilon|\log x|}\left|\frac{\frac{U^{\prime}(t x)}{t^{-1} a_{0}(t)}-x^{\gamma-1}}{\tilde{A}(t)}-\frac{d}{d x}\left(K_{\gamma, \rho}(x)\right)\right|<\varepsilon .
$$

For example, the function $a_{0}$ could be chosen as

$$
a_{0}(t)= \begin{cases}t U^{\prime}(t), & \gamma=\rho=0, \\ \gamma U(t), & \gamma>0=\rho, \\ -\gamma(U(\infty)-U(t)), & \gamma<0=\rho, \\ c t^{\gamma}, & \rho<0,\end{cases}
$$

with $c=\lim _{t \rightarrow \infty} t^{-\gamma} \tilde{a}(t)$ (which exists in that case). In the following we choose the function $a_{0}$ as defined in Eq. 2.6. Note also that, in Eq. 2.5 we may replace $\tilde{A}$ by any function $A_{*}$ such that $A_{*}(t) \sim \tilde{A}(t)$ for large $t$.

Now let us return to Eq. 2.4. The convergence of Eq. 2.4 is locally uniform, so by taking the integral on $[1, x]$ (or $[x, 1]$ ) for both sides of Eq. 2.4, it follows that as $t \rightarrow \infty$,

$$
\frac{\frac{U(t x)-U(t)}{\tilde{a}(t)}-\frac{x^{\gamma}-1}{\gamma}}{\tilde{A}(t)} \rightarrow K_{\gamma, \rho}(x) .
$$


Condition (2.7) is a popular second order condition in EVT. de Haan and Stadtmüller (1996) discuss the second order condition in details, which allowed for many asymptotic statistical results in EVT (see for example, Drees, 1998; Gomes and Martins, 2002; de Haan and Peng, 1998). Here we also present an uniformly weighted convergence of Eq. 2.7, which is similar to Eq. 2.5. In order to obtain this convergence, we have to replace also $\tilde{A}$ by a particular function $A_{0}$ in case of $\rho<0, \gamma+\rho \neq 0$, by defining

$$
A_{0}(t):= \begin{cases}(\gamma+\rho) a_{0}^{-1}(t) \bar{U}(t), & \text { if } \rho<0, \gamma+\rho>0, \\ -(\gamma+\rho) a_{0}^{-1}(t)(\bar{U}(\infty)-\bar{U}(t)), & \text { if } \rho<0, \gamma+\rho<0, \\ \tilde{A}(t), & \text { else, }\end{cases}
$$

with $\bar{U}(t)=U(t)-c\left(t^{\gamma}-1\right) / \gamma$ for $\rho<0$, and $c=\lim _{t \rightarrow \infty} t^{-\gamma} \tilde{a}(t)$ (which exists in that case).

Corollary 2.1 Suppose Eq. 1.5 holds. Then for each $\varepsilon>0$, there exists a $t_{\varepsilon}>0$ such that for all $t, t x>t_{\varepsilon}$

$$
x^{-(\gamma+\rho)} e^{-\varepsilon|\log x|}\left|\frac{\frac{U(t x)-U(t)}{a_{0}(t)}-\frac{x^{\gamma}-1}{\gamma}}{A_{0}(t)}-K_{\gamma, \rho}(x)\right|<\varepsilon .
$$

Remark 2.1 The assertion of Corollary 2.1 is the same as in Cheng and Jiang (2001) but with different definitions of $a_{0}$ and $A_{0}$. Here we use a much simpler definition for $a_{0}$ in case of $\gamma=\rho=0$ and keep the same definition for other cases; and we also apply the same definition for $A_{0}$ in case of $\rho<0, \gamma+\rho \neq 0$, but use different definitions for other cases. Generally speaking, the functions $a_{0}$ and $A_{0}$ defined in this paper are much simpler than those in Cheng et al. (2001) in several cases. On the other hand, by the definition of $K_{\gamma, \rho}$ it follows that if Eq. 2.9 holds and we replace $A_{0}$ by any asymptotically equivalent function $A_{*}$, then Eq. 2.9 still holds except in case $\rho<0, \gamma+\rho \neq 0$. This is the reason why we still keep the same definition of $A_{0}$ as in Cheng et al. (2001) in that case.

For each $\delta, c>0$ define

$$
D_{t, \rho}:=D_{t, \rho, \delta, c}:= \begin{cases}\left\{x:(1+\gamma x)^{-1 / \gamma} \leq c t^{-\delta+1}\right\}, & \text { if } \rho<0, \\ \left\{x:(1+\gamma x)^{-1 / \gamma} \leq\left|A_{0}(t)\right|^{-c}\right\}, & \text { if } \rho=0 .\end{cases}
$$


Corollary 2.2 Suppose Eq. 1.5 holds. Then for all $\varepsilon, \delta, c>0$

$$
\begin{aligned}
\sup _{x \in D_{t, \rho}} w_{F}(t, x) \mid & \frac{t \bar{F}\left(a_{0}(t) x+b_{0}(t)\right)-(1+\gamma x)^{-1 / \gamma}}{A_{0}(t)} \\
& -(1+\gamma x)^{-\frac{1}{\gamma}-1} \tilde{K}_{\gamma, \rho}\left((1+\gamma x)^{1 / \gamma}\right) \mid \rightarrow 0
\end{aligned}
$$

as $t \rightarrow \infty$, where

$$
\begin{array}{r}
b_{0}(t):= \begin{cases}U(t)-a_{0}(t) A_{0}(t) /(\gamma+\rho), & \text { if } \rho<0, \gamma+\rho \neq 0, \\
U(t), & \text { else, }\end{cases} \\
\tilde{K}_{\gamma, \rho}(x):= \begin{cases}K_{\gamma, \rho}(x)+\frac{1}{\gamma+\rho}, & \text { if } \rho<0, \gamma+\rho \neq 0, \\
K_{\gamma, \rho}(x), & \text { else, }\end{cases}
\end{array}
$$

and

$w_{F}(t, x):= \begin{cases}(1+\gamma x)^{-\frac{1}{\gamma}(\rho-1)} \exp \left(-\varepsilon\left|\log \left((1+\gamma x)^{-1 / \gamma}\right)\right|\right), & \gamma \neq 0 \text { or } \rho<0, \\ \min \left\{\left(t \bar{F}\left(a_{0}(t) x+b_{0}(t)\right)\right)^{-1} e^{-\varepsilon \log \left|t \bar{F}\left(a_{0}(t) x+b_{0}(t)\right)\right|}, e^{x-\varepsilon|x|}\right\}, & \gamma=\rho=0 .\end{cases}$

Corollary 2.3 Suppose Eq. 1.5 holds with $\gamma=\rho=0$. Then for all $\varepsilon, c>0$

$$
\sup _{\left\{x:\left|A_{0}(t)\right|^{c} \leq e^{-x} \leq\left|A_{0}(t)\right|^{-c}\right\}} e^{x-\varepsilon|x|}\left|\frac{t \bar{F}\left(a_{0}(t) x+b_{0}(t)\right)-e^{-x}}{A_{0}(t)}-e^{-x} \frac{x^{2}}{2}\right|=o(1) .
$$

Remark 2.2 The assertion of Corollary 2.2 is the same as that of Proposition 3.2 in Drees et al. (2006), but the conditions are much stronger and the definitions of $a_{0}$ and $A_{0}$ are different. That proposition is derived fully based on Eq. 2.9, where the functions $a_{0}, b_{0}$ and $A_{0}$ are not restricted as in our setup. So Corollary 2.1 implies Corollary 2.2. In case of $\gamma=\rho=0$, the two function $t \bar{F}\left(a_{0}(t) x+b_{0}(t)\right)$ and $e^{-x}$ can behave quite differently for sufficiently large $x$. But from the proof of Proposition 3.2 in Drees et al. (2006), we see

$$
\sup _{\left\{x:\left|A_{0}(t)\right|^{c} \leq t \bar{F}\left(a_{0}(t) x+b_{0}(t)\right) \leq\left|A_{0}(t)\right|^{-c}\right\}}\left|\frac{e^{-x}}{t \bar{F}\left(a_{0}(t) x+b_{0}(t)\right)}-1\right|=o(1) .
$$

Hence Corollary 2.3 follows by Corollary 2.2.

Based on Proposition 2.1, Corollary 2.1 and Corollary 2.2 we get our main results. 
Theorem 2.1 Suppose Eq. 1.5 holds. Then for each $\varepsilon, \delta, c>0$

$$
\sup _{x \in D_{t, \rho}} w_{f}(t, x)\left|\frac{t a_{0}(t) f\left(a_{0}(t) x+b_{0}(t)\right)-(1+\gamma x)^{-1-1 / \gamma}}{A_{0}(t)}+\mathrm{d}(\mathrm{x})\right| \rightarrow 0
$$

as $t \rightarrow \infty$, where $f$ is the density function of $F$,

$$
\begin{aligned}
d(x) & :=\frac{d}{\mathrm{~d} x}\left((1+\gamma x)^{-1 / \gamma-1} \tilde{K}_{\gamma, \rho}\left((1+\gamma x)^{1 / \gamma}\right)\right) \\
& = \begin{cases}\frac{\rho-1}{\gamma+\rho}(1+\gamma x)^{-\frac{1}{\gamma}(1-\rho)-1}, & \rho<0, \gamma+\rho \neq 0, \\
(1+\gamma x)^{-\frac{1}{\gamma}-2}\left(1-(1+\gamma) \log \left((1+\gamma x)^{1 / \gamma}\right)\right), & \rho<0, \gamma+\rho=0, \\
(1+\gamma x)^{-\frac{1}{\gamma}-1}\left(\frac{1}{\gamma}-\frac{1}{\gamma} \log \left((1+\gamma x)^{1 / \gamma}\right)\right), & \rho=0 \neq \gamma, \\
e^{-x}\left(x-\frac{x^{2}}{2}\right), & \rho=0=\gamma,\end{cases}
\end{aligned}
$$

and

$$
w_{f}(t, x):= \begin{cases}(1+\gamma x)^{-\frac{1}{\gamma}(\rho-1)+1} \exp \left(-\varepsilon\left|\log \left((1+\gamma x)^{-1 / \gamma}\right)\right|\right), & \gamma \neq 0 \text { or } \rho<0, \\ \min \left\{\frac{e^{-\varepsilon\left|\log \left(t a_{0}(t) f\left(a_{0}(t) x+b_{0}(t)\right)\right)\right|}}{t a_{0}(t) f\left(a_{0}(t) x+b_{0}(t)\right)}, e^{x-\varepsilon|x|}\right\}, & \gamma=\rho=0 .\end{cases}
$$

Corollary 2.4 Suppose Eq. 1.5 holds with $\gamma=\rho=0$. Then for each $\varepsilon, c>0$

$$
\sup _{\left\{x:\left|A_{0}(t)\right|^{c} \leq e^{-x} \leq\left|A_{0}(t)\right|^{-c}\right\}} e^{x-\varepsilon|x|}\left|\frac{t a_{0}(t) f\left(a_{0}(t) x+b_{0}(t)\right)-e^{-x}}{A_{0}(t)}+e^{-x}\left(x-\frac{x^{2}}{2}\right)\right|=o(1) .
$$

In Theorem 2.1 the weight function $w_{f}(t, x)$ in case of $\gamma=\rho=0$ is rather different to the function in other cases. The two functions $t a_{0}(t) f\left(a_{0}(t) x+\right.$ $\left.b_{0}(t)\right)$ and $e^{-x}$ behave differently for sufficiently large $x$, which implies that the minimum can not be replaced by any of the two functions. For more details see Drees et al. (2006). The proof of Corollary 2.4 will be presented in the proof of Theorem 2.1.

Now consider Eq. 1.3 for $L=1$. Theorem 2.1 gives an approximation to the tail density function of the underlying distribution. From Corollary 2.2 we can obtain an approximation to the tail distribution function of the normalized maximum (see Lemma 3.2 below). Based on the two approximations we derive the approximation to the tail density function of the normalized maximum. 
Theorem 2.2 Suppose Eq. 1.5 holds and that $\rho>-1$ but not $\gamma=\rho=0$. Then

$$
\begin{aligned}
\sup _{\left\{x:(1+\gamma x)^{-1 / \gamma} \leq \log ^{2}\left|A_{0}(n)\right|\right\}} w(x) & \times \mid \frac{\frac{d}{d x} F^{n}\left(a_{n} x+b_{n}\right)-\frac{d}{d x} G_{\gamma}(x)}{A_{0}(n)} \\
& +G_{\gamma}(x)\left((1+\gamma x)^{-2-2 / \gamma} \tilde{K}_{\gamma, \rho}\left((1+\gamma x)^{1 / \gamma}\right)+d(x)\right) \mid \rightarrow 0,
\end{aligned}
$$

as $n \rightarrow \infty$, where $a_{n}=a_{0}(n), b_{n}=b_{0}(n)$ and

$$
w(x)=\min \left\{w_{f}(n, x) G_{\gamma}^{-1}(x), \max \left\{1, w_{F}(n, x)\right\}(1+\gamma x)^{1+1 / \gamma}\right\} .
$$

Moreover, for any constant $x_{0} \in\left(-\frac{1}{\gamma \vee 0}, \frac{1}{(-\gamma) \vee 0}\right)$, as $n \rightarrow \infty$,

$$
\begin{gathered}
\sup _{x_{0} \leq x<\frac{1}{(-\gamma) \vee 0}}(1+\gamma x)^{-\frac{1}{\gamma}(\rho-1+\varepsilon)+1} \\
\times \mid \frac{\frac{d}{d x} F^{n}\left(a_{n} x+b_{n}\right)-\frac{d}{d x} G_{\gamma}(x)}{A_{0}(n)} \\
+G_{\gamma}(x)\left((1+\gamma x)^{-2-2 / \gamma} \tilde{K}_{\gamma, \rho}\left((1+\gamma x)^{1 / \gamma}\right)\right. \\
+d(x)) \mid \rightarrow 0 .
\end{gathered}
$$

Corollary 2.5 Suppose Eq. 1.5 holds with $\gamma=\rho=0$. Then

$$
\begin{aligned}
& \sup _{\left\{x: \log ^{-2}\left|A_{0}(n)\right| \leq e^{-x} \leq \log ^{2}\left|A_{0}(n)\right|\right\}} \min \left\{e^{x-\varepsilon|x|} e^{e^{-x}}, \max \left\{1, e^{2 x-\varepsilon|x|}\right\}\right\} \times \\
& \left|\frac{\frac{d}{d x} F^{n}\left(a_{n} x+b_{n}\right)-\frac{d}{d x} G_{0}(x)}{A_{0}(n)}+G_{0}(x)\left(e^{-2 x} \frac{x^{2}}{2}+e^{-x}\left(x-\frac{x^{2}}{2}\right)\right)\right|=o(1) .
\end{aligned}
$$

\section{Proofs}

Before proving the main results, we state a simple lemma on regular varying function.

Lemma 3.1 If $h \in \operatorname{RV}(\gamma)$ with $\gamma \in \mathbb{R}$, then for each $\varepsilon>0$ and $h^{*} \sim h$, there exists a $t_{\varepsilon}$ such that for all $t, t x \geq t_{\varepsilon}$,

$$
x^{-\gamma} e^{-\varepsilon|\log x|}\left|\frac{h(t x)}{h^{*}(t)}-x^{\gamma}\right| \leq \varepsilon .
$$

Proof Note that

$$
x^{-\gamma} e^{-\varepsilon|\log x|}\left|\frac{h(t x)}{h^{*}(t)}-x^{\gamma}\right| \leq x^{-\gamma} e^{-\varepsilon|\log x|}\left|\frac{h(t x)}{h(t)}-x^{\gamma}\right| \frac{h(t)}{h^{*}(t)}+e^{-\varepsilon|\log x|}\left|\frac{h(t)}{h^{*}(t)}-1\right| .
$$


So, as $\min \{t, t x\} \rightarrow \infty$, the first part converges to zero by Proposition 2.1 in Cheng et al. (2001) and $h^{*} \sim h$, and the second part converges to zero obviously. Hence the statement follows.

For simplicity, we denote $x^{ \pm \varepsilon}$ for $e^{-\varepsilon|\log x|}$ and $x^{\mp \varepsilon}$ for $e^{\varepsilon|\log x|}$ with $\varepsilon>0$. Note that for $x>0,0<x^{ \pm \varepsilon} \leq 1$ and $x^{\mp \varepsilon} \geq 1$.

Proof of Proposition 2.1 We distinguish the three cases: $\rho<0, \rho=0 \neq \gamma$ and $\rho=\gamma=0$.

(a) $\quad \rho<0$. Let $c=\lim _{t \rightarrow \infty} t^{-\gamma} \tilde{a}(t)$. By the definitions of $\tilde{a}$ and $\tilde{A}$ and by Eq. 2.1 it follows that

$$
\begin{aligned}
c & =\lim _{t \rightarrow \infty} t^{-\gamma} t U^{\prime}(t)\left(1-\frac{A(t)}{\rho}\right) \\
& =\lim _{t \rightarrow \infty} t^{-\gamma+1} k t^{\gamma-1} \exp \left(\int_{1}^{t} \frac{A(u)}{u} d u\right)\left(1-\frac{A(t)}{\rho}\right) \\
& =k \exp \left(\int_{1}^{\infty} \frac{A(u)}{u} d u\right) .
\end{aligned}
$$

Then $a_{0}(t)=c t^{\gamma}$ and for large $t$ and large $t x$

$$
\begin{aligned}
\frac{U^{\prime}(t x)}{t^{-1} a_{0}(t)}-x^{\gamma-1} & =\frac{k(t x)^{\gamma-1} \exp \left(\int_{1}^{t x} \frac{A(u)}{u} d u\right)}{t^{\gamma-1} k \exp \left(\int_{1}^{\infty} \frac{A(u)}{u} d u\right)}-x^{\gamma-1} \\
& =x^{\gamma-1}\left(\exp \left(-\int_{t x}^{\infty} \frac{A(u)}{u} d u\right)-1\right) \\
& =x^{\gamma-1}\left(-\int_{t x}^{\infty} \frac{A(u)}{u} d u(1+o(1))\right),
\end{aligned}
$$

since $\int_{t}^{\infty} A(u) u^{-1} d u \in \mathrm{RV}(\rho)$ with $\rho<0$. Note that $-\int_{t}^{\infty} \frac{A(u)}{u} d u \sim \rho^{-1}$ $A(t)$ as $t \rightarrow \infty$ (see e.g., Bingham et al., 1987) and that

$$
\begin{gathered}
x^{-(\gamma+\rho-1) \pm \varepsilon}\left|\frac{\frac{U^{\prime}(t x)}{t^{-1} a_{0}(t)}-x^{\gamma-1}}{\tilde{A}(t)}-x^{\gamma+\rho-1}\right| \\
=x^{-\rho \pm \varepsilon}\left|\frac{-\int_{t x}^{\infty} \frac{A(u)}{u} d u(1+o(1))}{A(t) / \rho}-x^{\rho}\right| \\
=x^{-\rho \pm \varepsilon}\left|\frac{(1+o(1)) A(t x)}{A(t)}-x^{\rho}\right| .
\end{gathered}
$$

Thus the statement follows by Lemma 3.1 in case of $\rho<0$. 
(b) $\rho=0 \neq \gamma$. First consider the case: $\gamma>0=\rho$. Note that

$$
\begin{aligned}
x^{-(\gamma-1) \pm \varepsilon}\left|\frac{\frac{U^{\prime}(t x)}{t^{-1} a_{0}(t)}-x^{\gamma-1}}{\tilde{A}(t)}-\left(x^{\gamma-1} \log x+\frac{x^{\gamma-1}}{\gamma}\right)\right| \\
=\gamma x^{-\gamma \pm \varepsilon}\left|\frac{\frac{(t x) U^{\prime}(t x)}{\gamma a_{0}(t)}-\frac{x^{\gamma}}{\gamma}}{\tilde{A}(t)}-\left(\frac{x^{\gamma} \log x}{\gamma}+\frac{x^{\gamma}}{\gamma^{2}}\right)\right| \\
\leq \gamma x^{-\gamma \pm \varepsilon}\left|\frac{\frac{U(t x)-U(t)}{a_{0}(t)}-\frac{x^{\gamma}-1}{\gamma}}{\tilde{A}(t)}-\frac{x^{\gamma} \log x}{\gamma}\right| \\
\quad+\gamma x^{-\gamma \pm \varepsilon}\left|\frac{\frac{(t x) U^{\prime}(t x)}{\gamma a_{0}(t)}-\frac{1}{\gamma}-\frac{U(t x)-U(t)}{a_{0}(t)}}{\tilde{A}(t)}-\frac{x^{\gamma}}{\gamma^{2}}\right| \\
=: I_{1}+I_{2} .
\end{aligned}
$$

Cheng et al. (2001) proved that $I_{1}=o(1)$ for $t$ and $t x$ large, so we only need to check that $I_{2}=o(1)$ for such $t$ and $t x$. In case of $\gamma>0=\rho, a_{0}(t)=$ $\gamma U(t), \tilde{A}(t)=A(t)$. Then

$$
I_{2}=\gamma x^{-\gamma \pm \varepsilon}\left|\frac{\frac{(t x) U^{\prime}(t x)}{\gamma^{2} U(t)}-\frac{U(t x)}{\gamma U(t)}}{A(t)}-\frac{x^{\gamma}}{\gamma^{2}}\right|=x^{-\gamma \pm \varepsilon}\left|\frac{\frac{(t x) U^{\prime}(t x)}{\gamma U(t)}-\frac{U(t x)}{U(t)}}{A(t)}-\frac{x^{\gamma}}{\gamma}\right| .
$$

Note that by Eq. 2.1 and by partial integration

$$
\begin{aligned}
U(t)-U(1)= & \int_{1}^{t} U^{\prime}(s) \mathrm{d} s=\int_{1}^{t} k s^{\gamma-1} \exp \left(\int_{1}^{s} \frac{A(u)}{u} d u\right) d s \\
= & \frac{1}{\gamma} \int_{1}^{t} k \exp \left(\int_{1}^{s} \frac{A(u)}{u} d u\right) d\left(s^{\gamma}\right) \\
= & \frac{1}{\gamma} k t^{\gamma} \exp \left(\int_{1}^{t} \frac{A(u)}{u} d u\right) \\
& -\frac{k}{\gamma}-\frac{1}{\gamma} \int_{1}^{t} k s^{\gamma-1} \exp \left(\int_{1}^{s} \frac{A(u)}{u} d u\right) A(s) d s .
\end{aligned}
$$

Thus

$$
\begin{aligned}
U(t) & =\frac{1}{\gamma} k t^{\gamma} \exp \left(\int_{1}^{t} \frac{A(u)}{u} d u\right)-\frac{k}{\gamma}-\frac{1}{\gamma} \int_{1}^{t} U^{\prime}(s) A(s) d s+U(1), \\
\gamma U(t x) & =k(t x)^{\gamma} \exp \left(\int_{1}^{t x} \frac{A(u)}{u} d u\right)-k-\int_{1}^{t x} U^{\prime}(s) A(s) d s+\gamma U(1), \\
(t x) U^{\prime}(t x) & =k(t x)^{\gamma} \exp \left(\int_{1}^{t x} \frac{A(u)}{u} d u\right) .
\end{aligned}
$$


Let $g(t)=U^{\prime}(t) A(t)$. Note that $\int_{1}^{t} g(s) d s \in \mathrm{RV}(\gamma)$ and $\int_{1}^{t} g(s) d s /\left(t U^{\prime}\right.$ $(t)) \rightarrow 0$ by using $g \in \mathrm{RV}(\gamma-1)$ with $\gamma>0$ and

$$
\operatorname{tg}(t) \sim \gamma \int_{1}^{t} g(s) d s, \quad \text { as } t \rightarrow \infty .
$$

Thus the numerator of the first term in Eq. 3.1 becomes

$$
\begin{aligned}
\frac{(t x) U^{\prime}(t x)}{\gamma U(t)}-\frac{U(t x)}{U(t)} & =\frac{(t x) U^{\prime}(t x)-\gamma U(t x)}{\gamma U(t)} \\
& =\frac{k+\int_{1}^{t x} U^{\prime}(s) A(s) d s+\gamma U(1)}{k t^{\gamma} \exp \left(\int_{1}^{t} \frac{A(u)}{u} \mathrm{~d} u\right)-k-\int_{1}^{t} U^{\prime}(s) A(s) d s+\gamma U(1)} \\
& =\frac{\int_{1}^{t x} U^{\prime}(s) A(s) d s}{k t^{\gamma} \exp \left(\int_{1}^{t} \frac{A(u)}{u} d u\right)}(1+o(1)) \\
& =\frac{\int_{1}^{t x} g(s) d s}{\operatorname{tg}(t) / A(t)}(1+o(1)),
\end{aligned}
$$

using the definition of $g$. In order to prove $I_{2}=o(1)$, it is sufficient to prove that, for $t$ and $t x$ large,

$$
I_{3}:=x^{-\gamma \pm \varepsilon}\left|\frac{\int_{1}^{t x} g(s) \mathrm{d} s}{\operatorname{tg}(t)(1+o(1))}-\frac{x^{\gamma}}{\gamma}\right|=o(1) .
$$

By Eq. 3.2 and Lemma 3.1 it follows that $I_{3} \rightarrow 0$ as $\min \{t, t x\} \rightarrow \infty$. Thus the statement in case of $\gamma>0=\rho$ follows. In case of $\gamma<0=\rho$, the proof is similar.

(c) $\rho=\gamma=0$. In this case, $a_{0}(t)=t U^{\prime}(t)$ and $\tilde{A}(t)=A(t)$. Note that

$$
\begin{aligned}
t x U^{\prime}(t x)-t U^{\prime}(t) & =\int_{t}^{t x}\left(s U^{\prime \prime}(s)+U^{\prime}(s)\right) d s \\
& =t \int_{1}^{x}\left(\frac{t s U^{\prime \prime}(t s)}{U^{\prime}(t s)}+1\right) U^{\prime}(t s) d s=t \int_{1}^{x} A(t s) U^{\prime}(t s) d s
\end{aligned}
$$

hence

$$
\begin{aligned}
& \frac{\frac{U^{\prime}(t x)}{t^{-1} a_{0}(t)}-x^{-1}}{\tilde{A}(t)}-x^{-1} \log x=\frac{\frac{U^{\prime}(t x)}{U^{\prime}(t)}-x^{-1}}{A(t)}-x^{-1} \log x \\
& =x^{-1}\left(\frac{t x U^{\prime}(t x)-t U^{\prime}(t)}{t U^{\prime}(t) A(t)}-\log x\right)=x^{-1} \int_{1}^{x}\left(\frac{A(t s) U^{\prime}(t s)}{A(t) U^{\prime}(t)}-\frac{1}{s}\right) d s .
\end{aligned}
$$


Since $A(t) U^{\prime}(t) \in \mathrm{RV}(-1)$, by Lemma 3.1, for each $\varepsilon>0$, for large $t$ and large $t x$

$$
\begin{aligned}
x^{1 \pm \varepsilon} & \left|\frac{\frac{U^{\prime}(t x)}{t^{-1} a_{0}(t)}-x^{-1}}{\tilde{A}(t)}-x^{-1} \log x\right| \leq x^{ \pm \varepsilon} \int_{1}^{x}\left|\frac{A(t s) U^{\prime}(t s)}{A(t) U^{\prime}(t)}-\frac{1}{s}\right| d s \\
& =x^{ \pm \varepsilon} \int_{1}^{x} o(1) s^{-1} s^{\mp \varepsilon / 2} d s \leq x^{ \pm \varepsilon} o(1) x^{\mp \varepsilon / 2} \int_{1}^{x} s^{-1} d s \\
& =o(1) x^{ \pm \varepsilon / 2} \log x=o(1) .
\end{aligned}
$$

Thus the statement follows in case $\rho=\gamma=0$, and the proof is finished.

\section{Proof of Corollary 2.1}

(a) $\gamma=\rho=0$. Note that

$$
\begin{aligned}
\frac{\frac{U(t x)-U(t)}{a_{0}(t)}-\log x}{A_{0}(t)}-\frac{1}{2} \log ^{2} x & =\int_{1}^{x}\left(\frac{\frac{U^{\prime}(t s)}{t^{-1} a_{0}(t)}-s^{-1}}{A_{0}(t)}-s^{-1} \log s\right) d s \\
& =\int_{1}^{x} s^{-1} \int_{1}^{s}\left(\frac{A(t u) U^{\prime}(t u)}{A(t) U^{\prime}(t)}-\frac{1}{u}\right) d u d s .
\end{aligned}
$$

By Lemma 3.1 it follows that, for each $\varepsilon>0$, for large $t$ and large $t x$,

$$
\begin{aligned}
& e^{-\varepsilon|\log x|}\left|\frac{\frac{U(t x)-U(t)}{a_{0}(t)}-\log x}{A_{0}(t)}-\frac{1}{2} \log ^{2} x\right| \\
& \leq x^{ \pm \varepsilon} \int_{1}^{x} s^{-1} \int_{1}^{s}\left|\frac{A(t u) U^{\prime}(t u)}{A(t) U^{\prime}(t)}-\frac{1}{u}\right| d u d s \\
& \leq x^{ \pm \varepsilon} \int_{1}^{x} s^{-1} \int_{1}^{s} o(1) u^{-1} u^{\mp \varepsilon / 2} d u d s \leq x^{ \pm \varepsilon} o(1) x^{\mp \varepsilon / 2} \int_{1}^{x} s^{-1} \int_{1}^{s} u^{-1} d u d s \\
& =o(1) x^{ \pm \varepsilon / 2} \frac{1}{2} \log ^{2} x=o(1) .
\end{aligned}
$$

(b) The proofs of the other cases were done by Cheng et al. (2001).

Proof of Theorem 2.1 The proof is similar to those of Proposition 3.1 and Proposition 3.2 in Drees et al. (2006). Here we only sketch out the main difference. For technical details we refer to that paper.

Since $F(U(t))=1-1 / t$, it follows that

$$
f(U(t)) U^{\prime}(t)=t^{-2} .
$$

Replace $t$ in Eq. (3.4) by $U^{\leftarrow}\left(a_{0}(t) x+b_{0}(t)\right)=1 / \bar{F}\left(a_{0}(t) x+b_{0}(t)\right)$, then

$$
f\left(a_{0}(t) x+b_{0}(t)\right)=\frac{\bar{F}^{2}\left(a_{0}(t) x+b_{0}(t)\right)}{U^{\prime}\left(1 / \bar{F}\left(a_{0}(t) x+b_{0}(t)\right)\right)} .
$$


Define

$$
y:=y(t, x):=\frac{1}{t \bar{F}\left(a_{0}(t) x+b_{0}(t)\right)}, \quad z:=z(x):=(1+\gamma x)^{-1 / \gamma},
$$

and for each $\delta, c>0$

$$
\tilde{D}_{t, \rho}:=\tilde{D}_{t, \rho, \delta, c}:= \begin{cases}\left\{z: z \leq c t^{-\delta+1}\right\}, & \text { if } \rho<0, \\ \left\{z: z \leq\left|A_{0}(t)\right|^{-c}\right\}, & \text { if } \rho=0 .\end{cases}
$$

Then $x \in D_{t, \rho} \Leftrightarrow z \in \tilde{D}_{t, \rho}$. We distinguish again the three cases: $\rho<0, \rho=0 \neq \gamma$ and $\rho=\gamma=0$.

(a) $\rho<0$. First consider $\gamma+\rho \neq 0$. By Corollary 2.2 it follows that for each $\varepsilon>0$,

$$
\begin{aligned}
y^{-1} & =z+A_{0}(t) z^{\gamma+1} \tilde{K}_{\gamma, \rho}\left(z^{-1}\right)+o(1) A_{0}(t) z^{1-\rho} z^{\mp \varepsilon} \\
& =z+A_{0}(t) \frac{1}{\gamma+\rho} z^{1-\rho}+o(1) A_{0}(t) z^{1-\rho \mp \varepsilon}
\end{aligned}
$$

for large $t$ and uniformly for $z \in \tilde{D}_{t, \rho}$.By Eq. 3.6 it is not difficult to show that $t y \rightarrow \infty$ as $t \rightarrow \infty$ uniformly for $x \in D_{t, \rho}$. Now we can expand $U^{\prime}(1 /$ $\left.\bar{F}\left(a_{0}(t) x+b_{0}(t)\right)\right)$ by using Eq. 2.5. Then for large $t$ and $x \in D_{t, \rho}$,

$$
\begin{aligned}
U^{\prime}\left(1 / \bar{F}\left(a_{0}(t) x+b_{0}(t)\right)\right)= & U^{\prime}(t y) \\
= & t^{-1} a_{0}(t)\left(y^{\gamma-1}+A_{0}(t) K_{\gamma, \rho}^{\prime}(y)\right. \\
& \left.+o(1) A_{0}(t) y^{\gamma+\rho-1} y^{\mp \varepsilon}\right) \\
= & t^{-1} a_{0}(t) y^{\gamma-1}\left(1+A_{0}(t) y^{\rho}+o(1) A_{0}(t) y^{\rho \mp \varepsilon}\right),
\end{aligned}
$$

and hence using Eq. 3.5 and the definition of $y$,

$$
t a_{0}(t) f\left(a_{0}(t) x+b_{0}(t)\right)=\frac{y^{-\gamma-1}}{1+A_{0}(t) y^{\rho}+o(1) A_{0}(t) y^{\rho \mp \varepsilon}} .
$$

In order to expand $t a_{0}(t) f\left(a_{0}(t) x+b_{0}(t)\right)$ further, we show that

$$
A_{0}(t) y^{\rho \mp \varepsilon} \rightarrow 0 \text {, as } t \rightarrow \infty \text {, uniformly for } x \in D_{t, \rho} .
$$

If $y \geq 1$, then $A_{0}(t) y^{\rho \mp \varepsilon}=A_{0}(t) y^{\rho+\varepsilon} \rightarrow 0$ by only choosing $\varepsilon<-\rho$. Now suppose $0<y<1$, then $A_{0}(t) y^{\rho \mp \varepsilon}=A_{0}(t) y^{\rho-\varepsilon}$ and by Eq. 3.6

$$
y^{\rho-\varepsilon}=\left(y^{-1}\right)^{-\rho+\varepsilon}=z^{-\rho+\varepsilon}\left(1+\frac{A_{0}(t)}{\gamma+\rho} z^{-\rho}+o(1) A_{0}(t) z^{-\rho \mp \varepsilon}\right)^{-\rho+\varepsilon} .
$$

For simplicity we assume here that $A_{0}(t)=t^{\rho}$ (in case of $A_{0}(t)=t^{\rho} l(t)$ with $l \in \mathrm{RV}(0)$, the proof is similar). For $z \in \tilde{D}_{t, \rho}$,

$A_{0}(t) z^{-\rho+\varepsilon} \leq t^{\rho}\left(c t^{-\delta+1}\right)^{-\rho+\varepsilon}=c^{-\rho+\varepsilon} t^{\delta \rho-\delta \varepsilon+\varepsilon} \rightarrow 0, \quad$ (for sufficient small $\varepsilon>0$ ) 
and also $A_{0}(t) z^{-\rho-\varepsilon} \rightarrow 0$ as $t \rightarrow \infty$ and uniformly for $z \in \tilde{D}_{t, \rho}$. Thus Eq. 3.8 holds and by expanding Eq. 3.7 and using Eq. 3.6,

$$
\begin{aligned}
t a_{0}(t) f( & \left.a_{0}(t) x+b_{0}(t)\right)=y^{-(\gamma+1)}\left(1-A_{0}(t) y^{\rho}+o(1) A_{0}(t) y^{\rho} y^{\mp \varepsilon}\right) \\
= & \left(z\left(1+\frac{A_{0}(t)}{\gamma+\rho} z^{-\rho}+o(1) A_{0}(t) z^{-\rho \mp \varepsilon}\right)\right)^{\gamma+1} \\
& -A_{0}(t)\left(z\left(1+\frac{A_{0}(t)}{\gamma+\rho} z^{-\rho}+o(1) A_{0}(t) z^{-\rho \mp \varepsilon}\right)\right)^{\gamma-\rho+1} \\
& +o(1) A_{0}(t)\left(z\left(1+\frac{A_{0}(t)}{\gamma+\rho} z^{-\rho}+o(1) A_{0}(t) z^{-\rho \mp \varepsilon}\right)\right)^{\gamma-\rho+1 \mp \varepsilon} \\
= & z^{\gamma+1}\left(1+\frac{1+\gamma}{\gamma+\rho} A_{0}(t) z^{-\rho}+o(1) A_{0}(t) z^{-\rho \mp \varepsilon}\right) \\
& -A_{0}(t) z^{\gamma-\rho+1}+o(1) A_{0}(t) z^{\gamma-\rho+1 \mp \varepsilon} \\
= & z^{\gamma+1}+\frac{1-\rho}{\gamma+\rho} A_{0}(t) z^{\gamma-\rho+1}+o(1) A_{0}(t) z^{\gamma-\rho+1 \mp \varepsilon}
\end{aligned}
$$

as $t \rightarrow \infty$, uniformly for $x \in D_{t, \rho}$. Hence Eq. 2.11 holds for $\rho<0$ and $\gamma+\rho \neq 0$. In case of $\rho<0$ and $\gamma+\rho=0$, the proof is similar.

(b) $\rho=0 \neq \gamma$. Let

$$
B(t, x)=\left|\frac{t a_{0}(t) f\left(a_{0}(t) x+b_{0}(t)\right)-(1+\gamma x)^{-\frac{1}{\gamma}-1}}{A_{0}(t)}+\mathrm{d}(x)\right|
$$

and recall

$$
w_{f}(t, x)=w_{f}(\varepsilon, t, x)=(1+\gamma x)^{1+1 / \gamma} \exp \left(-\varepsilon\left|\log \left((1+\gamma x)^{-1 / \gamma}\right)\right|\right) .
$$

For fixed $\varepsilon_{0}>0$ and $c_{0}>0$, $\sup _{\left\{x:(1+\gamma x)^{-1 / \gamma} \leq\left|A_{0}(t)\right|^{\left.-c_{0}\right\}}\right.} w_{f}\left(\varepsilon_{0}, t, x\right) B(t, x) \rightarrow 0$ as $t \rightarrow \infty$ is implied by showing

$$
\sup _{\left\{x:(1+\gamma x)^{-1 / \gamma} \leq\left|A_{0}(t)\right|^{c}\right\}} w_{f}\left(\varepsilon_{0}, t, x\right) B(t, x) \rightarrow 0
$$

with $c \geq c_{0}$ and showing

$$
\sup _{\left\{x:\left|A_{0}(t)\right|^{c} \leq(1+\gamma x)^{-1 / \gamma} \leq\left|A_{0}(t)\right|^{-c}\right\}} w_{f}(\varepsilon, t, x) B(t, x) \rightarrow 0
$$

with $\varepsilon \leq \varepsilon_{0}\left(\right.$ since $w_{f}\left(\varepsilon_{0}, t, x\right) \leq w_{f}(\varepsilon, t, x)$ ). Thus, in order to prove relation 2.11, we need to check that for fixed $\varepsilon>0$ and sufficiently large $c>0$

$$
\sup _{\left\{x:(1+\gamma x)^{-1 / \gamma} \leq\left|A_{0}(t)\right|^{c}\right\}} w_{f}(t, x) B(t, x) \rightarrow 0
$$


and that for fixed $c>0$ and sufficiently small $\varepsilon>0$

$$
\sup _{\left\{x:\left|A_{0}(t)\right|^{c} \leq(1+\gamma x)^{-1 / \gamma} \leq\left|A_{0}(t)\right|^{-c}\right\}} w_{f}(t, x) B(t, x) \rightarrow 0 .
$$

First consider Eq. 3.9. Note that

$$
\left.w_{f}(t, x)(1+\gamma x)^{-1 / \gamma-1}=(1+\gamma x)^{-\varepsilon / \gamma} \leq\left|A_{0}(t)\right|^{c \varepsilon}=o\left(A_{0}(t)\right), \quad \text { (if } c>1 / \varepsilon\right)
$$

for $x \in\left\{x:(1+\gamma x)^{-1 / \gamma} \leq\left|A_{0}(t)\right|^{c}\right\}$, and

$$
w_{f}(t, x) d(x)=(1+\gamma x)^{-\varepsilon / \gamma}\left(\frac{1}{\gamma}-\frac{1}{\gamma} \log \left((1+\gamma x)^{1 / \gamma}\right)\right) \rightarrow 0
$$

as $t \rightarrow \infty$ and uniformly for $x \in\left\{x:(1+\gamma x)^{-1 / \gamma} \leq\left|A_{0}(t)\right|^{c}\right\}$. So for Eq. 3.9, it remains to prove that

$$
\sup _{\left\{x:(1+\gamma x)^{-1 / \gamma} \leq\left|A_{0}(t)\right|^{c}\right\}} w_{f}(t, x) t a_{0}(t) f\left(a_{0}(t) x+b_{0}(t)\right)=o\left(A_{0}(t)\right) .
$$

Suppose $\gamma>0$. Note that $a_{0}(t) x+b_{0}(t)=(1+\gamma x) U(t) \rightarrow \infty$ uniformly for $x \in\left\{x:(1+\gamma x)^{-1 / \gamma} \leq\left|A_{0}(t)\right|^{c}\right\}$. Relation 1.4 implies that

$$
\frac{f((1+\gamma x) U(t))}{f(U(t))}=\frac{t a_{0}(t) f\left(a_{0}(t) x+b_{0}(t)\right)}{t a_{0}(t) f\left(b_{0}(t)\right)} \rightarrow(1+\gamma x)^{-1-1 / \gamma} .
$$

Thus $f \in \mathrm{RV}(-1-1 / \gamma)$. By the Potter bounds for regular varying function it follows that by Eq. 3.4

$$
t^{2} U^{\prime}(t) f\left(a_{0}(t) x+b_{0}(t)\right)=\frac{f((1+\gamma x) U(t))}{f(U(t))} \leq 2(1+\gamma x)^{\left(-1 / \gamma-1+\varepsilon_{1} / \gamma\right)},
$$

choosing $\varepsilon_{1}=\varepsilon / 2$. By Eq. 2.5 one has

$$
U^{\prime}(t)=t^{-1} a_{0}(t)\left(1+A_{0}(t) / \gamma+o(1) A_{0}(t)\right),
$$

thus

$$
t a_{0}(t) f\left(a_{0}(t) x+b_{0}(t)\right) \leq \frac{2(1+\gamma x)^{\left(-1 / \gamma-1+\varepsilon_{1} / \gamma\right)}}{1+A_{0}(t) / \gamma+o\left(A_{0}(t)\right)} .
$$

Hence for large $t$

$$
\begin{aligned}
w_{f}(t, x) t a_{0}(t) f\left(a_{0}(t) x+b_{0}(t)\right) & \leq 4(1+\gamma x)^{-\frac{\varepsilon}{2 \gamma}} \\
& \leq 4\left|A_{0}(t)\right|^{\frac{c \varepsilon}{2}}=o\left(A_{0}(t)\right), \quad \text { if } c>2 / \varepsilon .
\end{aligned}
$$

In case of $\gamma<0$ the steps are similar. Hence Eq. 3.9 holds.

Now consider Eq. 3.10. By Corollary 2.2 and Corollary 2.3, for each $\varepsilon>0$

$$
\begin{aligned}
y^{-1} & =z+A_{0}(t) z^{\gamma+1} \tilde{K}_{\gamma, 0}\left(z^{-1}\right)+o(1) A_{0}(t) z z^{\mp \varepsilon} \\
& =z-A_{0}(t) \frac{1}{\gamma} z \log z+o(1) A_{0}(t) z^{1 \mp \varepsilon}
\end{aligned}
$$


for large $t$ and uniformly for $z \in \tilde{D}_{t, 0}$. Again, we obtain that $t y \rightarrow \infty$ as $t \rightarrow \infty$ uniformly for $x \in D_{t, 0}$. As in Eq. 3.7, it follows that

$$
U^{\prime}\left(1 / \bar{F}\left(a_{0}(t) x+b_{0}(t)\right)\right)=t^{-1} a_{0}(t) y^{\gamma-1}\left(1+A_{0}(t)\left(\log y+\frac{1}{\gamma}\right)+o(1) A_{0}(t) y^{\mp \varepsilon}\right)
$$

and

$$
t a_{0}(t) f\left(a_{0}(t) x+b_{0}(t)\right)=\frac{y^{-(\gamma+1)}}{1+A_{0}(t)\left(\log y+\frac{1}{\gamma}\right)+o(1) A_{0}(t) y^{\mp \varepsilon}}
$$

for large $t$ and uniformly for $x \in D_{t, 0}$.

As in case (a), it follows that forciently small $\varepsilon>0, A_{0}(t) y^{\mp \varepsilon} \rightarrow 0$ as $t \rightarrow \infty$ and uniformly for $x \in\left\{x:\left|A_{0}(t)\right|^{c} \leq(1+\gamma x)^{-1 / \gamma} \leq\left|A_{0}(t)\right|^{-c}\right\}$ hence $A(t) \log z \rightarrow 0$, and that

$$
t a_{0}(t) f\left(a_{0}(t) x+b_{0}(t)\right)=z^{\gamma+1}-A_{0}(t) z^{\gamma+1}\left(\frac{1}{\gamma}+\frac{1}{\gamma} \log z\right)+o(1) A_{0}(t) z^{\gamma+1 \mp \varepsilon}
$$

as $t \rightarrow \infty$, uniformly for $x \in\left\{x:\left|A_{0}(t)\right|^{c} \leq(1+\gamma x)^{-1 / \gamma} \leq\left|A_{0}(t)\right|^{-c}\right\}$.

Hence Eq. 3.10 holds.

(c) $\rho=\gamma=0$. By Corollary 2.3 we have that for large $t$ and

$x \in\left\{x:\left|A_{0}(t)\right|^{c} \leq e^{-x} \leq\left|A_{0}(t)\right|^{-c}\right\}$

$$
y^{-1}=e^{-x}+A_{0}(t) e^{-x} \frac{x^{2}}{2}+o(1) A_{0}(t) e^{-x+\varepsilon|x|} .
$$

Arguing as in case (b), we have

$$
\sup _{\left\{x:\left|A_{0}(t)\right|^{c} \leq e^{-x} \leq\left|A_{0}(t)\right|^{-c}\right\}} e^{x-\varepsilon|x|}\left|\frac{\mid a_{0}(t) f\left(a_{0}(t) x+b_{0}(t)\right)-e^{-x}}{A_{0}(t)}+e^{-x}\left(x-\frac{x^{2}}{2}\right)\right| \rightarrow 0
$$

as $t \rightarrow \infty$. Hence Corollary 2.4 follows. Now consider the set $\left\{x: e^{-x} \leq\left|A_{0}(t)\right|^{c}\right\}$. Since

$$
e^{x-\varepsilon|x|} \cdot e^{-x}\left(x-\frac{x^{2}}{2}\right) \rightarrow 0, \quad e^{x-\varepsilon|x|} \cdot e^{-x} / A_{0}(t) \leq\left|A_{0}(t)\right|^{\varepsilon c-1} \rightarrow 0, \quad(\text { if } c>1 / \varepsilon)
$$

it is sufficient for Eq. 2.11 to check

$$
\sup _{\left\{x: e^{-x} \leq \mid A_{0}(t)^{c}\right\}} \frac{\exp \left(-\varepsilon\left|\log \left(t a_{0}(t)\right) f\left(a_{0}(t) x+b_{0}(t)\right)\right|\right.}{A_{0}(t)} \rightarrow 0
$$

as $t \rightarrow \infty$. Let $x_{t}=-c \log \left|A_{0}(t)\right|$. By Eq. 3.12 it follows

$$
\begin{gathered}
t a_{0}(t) f\left(a_{0}(t) x_{t}+b_{0}(t)\right)=e^{-x_{t}}-A_{0}(t) e^{-x_{t}}\left(x_{t}-\frac{x_{t}^{2}}{2}\right)+o(1) A_{0}(t) e^{-x_{t}(1-\varepsilon)} \\
\leq \max \left\{e^{-x_{t}},\left|A_{0}(t)\right| e^{-x_{t}(1-\varepsilon)}\right\}=\max \left\{\left|A_{0}(t)\right|^{c},\left|A_{0}(t)\right|^{c(1-\varepsilon)+1}\right\},
\end{gathered}
$$


for large $t$. By Eq. 2.1 and Eq. 3.4, it follows that $f(U(t))=k^{-1} \exp \left(-\int_{1}^{t} \frac{1+A(u)}{u}\right.$ $d u$ ) with $k>0$, which implies by taking the derivative that $f$ is decreasing near the right endpoint of $F$ since $A(t) \rightarrow 0$. Hence for $x \in\left\{x: e^{-x} \leq\left|A_{0}(t)\right|^{c}\right\}$, one has $x \geq x_{t}$ and

$$
\begin{aligned}
& \frac{\left(t a_{0}(t) f\left(a_{0}(t) x+b_{0}(t)\right)\right)^{\varepsilon}}{\left|A_{0}(t)\right|} \leq \frac{\left(t a_{0}(t) f\left(a_{0}(t) x_{t}+b_{0}(t)\right)\right)^{\varepsilon}}{\left|A_{0}(t)\right|} \\
& \quad \leq \max \left\{\left|A_{0}(t)\right|^{c \varepsilon-1},\left|A_{0}(t)\right|^{c \varepsilon(1-\varepsilon)+\varepsilon-1}\right\} \rightarrow 0, \quad(\text { if } c>2 / \varepsilon, \varepsilon<1 / 4)
\end{aligned}
$$

as $t \rightarrow \infty$. This finishes the proof of (c).

To prove Theorem 2.2, we need the following lemma.

Lemma 3.2 Suppose Eq. 1.5 holds. Then for each $\varepsilon>0$,

(a) for $\rho>-1$ but not $\gamma=\rho=0$,

$$
\begin{aligned}
& \sup _{\left\{x:(1+\gamma x)^{-1 / \gamma} \leq \log ^{2}\left|A_{0}(n)\right|\right\}} \max \left\{1, w_{F}(n, x)\right\} \times \\
& \left|\frac{F^{n-j}\left(a_{n} x+b_{n}\right)-G_{\gamma}(x)}{A_{0}(n)}+G_{\gamma}(x)(1+\gamma x)^{-1-1 / \gamma} \tilde{K}_{\gamma, \rho}\left((1+\gamma x)^{1 / \gamma}\right)\right|=o(1) ;
\end{aligned}
$$

(b) for $\gamma=\rho=0$,

$$
\begin{aligned}
& \sup _{\left\{x: \log ^{-2}\left|A_{0}(n)\right| \leq e^{-x} \leq \log ^{2}\left|A_{0}(n)\right|\right\}} \max \left\{1, e^{x-\varepsilon|x|}\right\} \\
& \times\left|\frac{F^{n-j}\left(a_{n} x+b_{n}\right)-G_{0}(x)}{A_{0}(n)}+G_{0}(x) e^{-x} \frac{x^{2}}{2}\right|=o(1) .
\end{aligned}
$$

Here $a_{n}=a_{0}(n), b_{n}=b_{0}(n)$, and $j$ is a fixed integer.

Proof (a) The proof is similar to that of Proposition 3.1 in Dress et al. (2003). Here we prove only the case $j=0$. Let

$$
\Phi(x)=(1+\gamma x)^{-1-1 / \gamma} \tilde{K}_{\gamma, \rho}\left((1+\gamma x)^{1 / \gamma}\right) .
$$

By Corollary 2.2, it follows that

$$
n \bar{F}\left(a_{n} x+b_{n}\right)=(1+\gamma x)^{-1 / \gamma}+A_{0}(n) \Phi(x)+o(1) \frac{A_{0}(n)}{w_{F}(n, x)}
$$


and $\bar{F}\left(a_{n} x+b_{n}\right) \rightarrow 0$ uniformly for all $x \in\left\{x:(1+\gamma x)^{-1 / \gamma} \leq \log ^{2}\left|A_{0}(n)\right|\right\}$. Hence it is immediate that

$$
\begin{aligned}
F^{n}\left(a_{n} x+b_{n}\right)= & \exp \left(-n \bar{F}\left(a_{n} x+b_{n}\right)\left(1+O\left(\bar{F}\left(a_{n} x+b_{n}\right)\right)\right)\right) \\
= & \left(\operatorname { e x p } \left(-(1+\gamma x)^{-1 / \gamma}-A_{0}(n) \Phi(x)\right.\right. \\
& \left.\left.+o(1) \frac{A_{0}(n)}{w_{F}(n, x)}\right)\right)^{1+O\left(\bar{F}\left(a_{n} x+b_{n}\right)\right)} \\
= & \left(G_{\gamma}(x)\left(1-A_{0}(n) \Phi(x)+o(1) \frac{A_{0}(n)}{w_{F}(n, x)}\right)\right)^{1+O\left(\bar{F}\left(a_{n} x+b_{n}\right)\right)} \\
= & G_{\gamma}(x) \exp \left(-(1+\gamma x)^{-1 / \gamma} O\left(\bar{F}\left(a_{n} x+b_{n}\right)\right)\right) \\
& \times\left(1-A_{0}(n) \Phi(x)+o(1) \frac{A_{0}(n)}{w_{F}(n, x)}\right)^{1+O\left(\bar{F}\left(a_{n} x+b_{n}\right)\right)} .
\end{aligned}
$$

Taking Taylor expansions to the last two factors, it follows that

$$
\begin{aligned}
F^{n}\left(a_{n} x+b_{n}\right)=G_{\gamma}(x)( & -A_{0}(n) \Phi(x)+o(1) \frac{A_{0}(n)}{w_{F}(n, x)} \\
& \left.-O(1) \frac{1}{n}(1+\gamma x)^{-2 / \gamma}+R_{n}(x)\right)
\end{aligned}
$$

and

$$
R_{n}(x)=o\left(\min \left\{\frac{A_{0}(n)}{w_{F}(n, x)}, \frac{1}{n}(1+\gamma x)^{-2 / \gamma}\right\}\right)
$$

uniformly for all $x \in\left\{x:(1+\gamma x)^{-1 / \gamma} \leq \log ^{2}\left|A_{0}(n)\right|\right\}$. If $\rho>-1$, then

$$
\frac{\frac{1}{n}(1+\gamma x)^{-2 / \gamma}}{A_{0}(n) / w_{F}(n, x)}=\frac{(1+\gamma x)^{-\frac{1}{\gamma}(1+\rho \pm \varepsilon)}}{n A_{0}(n)} \rightarrow 0 .
$$

Thus

$$
F^{n}\left(a_{n} x+b_{n}\right)=G_{\gamma}(x)\left(1-A_{0}(n) \Phi(x)+o(1) \frac{A_{0}(n)}{w_{F}(n, x)}\right)
$$

uniformly for all $x \in\left\{x:(1+\gamma x)^{-1 / \gamma} \leq \log ^{2}\left|A_{0}(n)\right|\right\}$, and hence Eq. 3.13 holds for $j=0$ since $\max \left\{1, w_{F}(n, x)\right\} G_{\gamma}(x) / w_{F}(n, x)$ is uniformly bounded in $x$. For any other integer $j$ the proof is similar. The proof of (b) is the same as that of (a).

Proof of Theorem 2.2 It follows that by Theorem 2.1,

$$
n a_{n} f\left(a_{n} x+b_{n}\right)=(1+\gamma x)^{-1-1 / \gamma}-A_{0}(n) d(x)+o(1) A_{0}(n) w_{f}^{-1}(n, x)
$$


and by Lemma 3.2,

$$
F^{n-1}\left(a_{n} x+b_{n}\right)=G_{\gamma}(x)-A_{0}(n) \Phi(x) G_{\gamma}(x)+o(1) A_{0}(n)\left(\max \left\{1, w_{F}(n, x)\right\}\right)^{-1}
$$

uniformly for all $x \in\left\{x:(1+\gamma x)^{-1 / \gamma} \leq \log ^{2}\left|A_{0}(n)\right|\right\}$. Here $a_{n}=a_{0}(n), b_{n}=$ $b_{0}(n)$ and $\Phi$ is defined as in Eq. 3.14. Thus multiplying the two formulas above, we get

$$
\begin{array}{rl}
n a_{n} & f\left(a_{n} x+b_{n}\right) F^{n-1}\left(a_{n} x+b_{n}\right) \\
= & G_{\gamma}(x)(1+\gamma x)^{-1-1 / \gamma}-A_{0}(n) G_{\gamma}(x)\left(\Phi(x)(1+\gamma x)^{-1-1 / \gamma}+\mathrm{d}(x)\right) \\
& +o(1) A_{0}(n)\left(G_{\gamma}(x) w_{f}^{-1}(n, x)+(1+\gamma x)^{-1-1 / \gamma}\left(\max \left\{1, w_{F}(n, x)\right\}\right)^{-1}\right)
\end{array}
$$

uniformly for all $x \in\left\{x:(1+\gamma x)^{-1 / \gamma} \leq \log ^{2}\left|A_{0}(n)\right|\right\}$. Hence Eq. 2.12 follows and Eq. 2.13 is immediate from Eq. 2.12.

\section{Proof of Corollary 2.5 The proof is similar to that of Theorem 2.2.}

Acknowledgements The authors thank Laurens de Haan for his comments on the proof of this paper and the referee for careful reading and valuable suggestion.

\section{References}

Bingham, N.H., Goldie, C.M., Teugels, J.L.: Regular Variation. Cambridge University Press, UK (1987)

Cheng, S., Jiang, C.: The edgeworth expansion for distributions of extreme values. Sci. China 44, 427-437 (2001)

Drees, H.: On smooth statistical tails functionals. Scand. J. Statist. 25, 187-210 (1998)

Drees, H., de Haan, L., Li, D: On large deviation for extremes. Stat. Probab. Lett. 64, 51-62 (2003)

Drees, H., de Haan, L., Li, D: Approximations to the tail empirical distribution function with application to testing extreme value conditions. J. Stat. Plan. Inference 136, 3498-3538 (2006)

Gomes, M.I., Martins, M.J.: Asymptotically unbiased estimators of the tail index based on external estimation of the second order parameters. Extremes 5, 5-31 (2002)

de Haan, L., Peng, L.: Comparison of tail index estimators. Statistica Neerlandica 52, 60-70 (1998)

de Haan, L., Resnick, S.I.: Local limit theorems for sample extremes. Ann. Probab. 10, 396-413 (1982)

de Haan, L., Resnick, S.I.: Second order regular variation and rates of convergence in extreme value theory. Ann. Probab. 24, 97-124 (1996)

de Haan, L., Stadtmüller, U: Generalized regular variation of second order. J. Austral. Math. Soc. A. Pure Math. 61, 381-395 (1996)

Hashorva, E.J.: On the number of near-maximum. Suppl. Rendic. Circ. Matemat. Palermo, Serie II, 65, 121-136 (2000)

Gabaix, X., Laibson, D.: Some Game Theory with Extreme Value Theory. Working Paper (2003)

Li, D., de Vries, C.L.: On the probability of being maximal(submitted, 2006)

Pickands, J.: The continuous and differentiable domains of attraction of the extreme value distributions. Ann. Probab. 14, 996-1004 (1986)

Rinott, Y., Rotar, V.: A remark on quadrant normal probabilities in high dimensions. Statist. Probab. Letters 51, 47-51 (2001)

Smith, R.L.: Uniform rates of convergence in extreme value theory. Adv. Appl. Probab. 14, 600$622(1982)$ 